[Article]

\title{
非血红素铁(III)活化氧分子反应的自旋轨道耦合和零场分裂
}

\author{
吕玲玲 ${ }^{1, *}$ 王小芳 ${ }^{1}$ 朱元成 ${ }^{1} \quad$ 刘新文 $^{1}$ 袁 焜 $^{1}$ 王永成 $^{2}$ \\ ('天水师范学院生命科学与化学学院, 甘肃天水 741001 ; 2 西北师范大学化学化工学院, 兰州 730070)
}

\begin{abstract}
摘要: 采用密度泛函理论对原儿茶酚 3,4-双加氧酶(3,4-PCD)活化 $\mathrm{O}_{2}$ 分子的反应机理进行了探讨. 初始复合 物, 六重态 ${ }^{6} 1$ 的超快形成主要归因于电子交换诱导系间穿越(EISC), $\mathrm{Fe} d_{z 2}: \mathrm{O}_{2} \pi^{*}(z)$ 是主要的交换通道, 在 $\mathrm{Fe}-$ $\mathrm{O}$ 键长为 $0.2487 \mathrm{~nm}$ 处, 交换重叠积分 $S_{i j}=\left\langle d_{z^{2}} \alpha \mid \pi^{*}(z) \beta\right\rangle=0.3758$. 从六重态 ${ }^{6} 1$ 形成四重态中间体 ${ }^{4} 1$, 有两种效 应共存, 即电子交换耦合作用和自旋轨道耦合(SOC)作用, 且相互竞争. 计算结果表明, 自旋轨道耦合(SOC)作 用起主导因素 $\left(S O C=353.16 \mathrm{~cm}^{-1}\right)$. 至于 $O-O$ 键的解离主要取决于儿茶酚 $(P C A)$ 最高占据分子轨道 $(H O M O)$ 的电子转移, 非血红素酶的铁中心仅承担 PCA 向 $\mathrm{O}_{2}$ 电子转移的缓冲作用.
\end{abstract}

关键词: 原儿茶酚 3,4-双加氧酶; 自旋轨道耦合; 零场分裂; 反应机理

中图分类号: $\mathrm{O} 641$

\section{Spin-Orbit Coupling and Zero-Field Splitting in Dioxygen Activation by Non-Heme Iron(III)}

\author{
LÜ Ling-Ling ${ }^{1, *} \quad$ WANG Xiao-Fang ${ }^{1} \quad$ ZHU Yuan-Cheng ${ }^{1} \quad$ LIU Xin-Wen ${ }^{1}$ \\ YUAN Kun ${ }^{1}$ WANG Yong-Cheng ${ }^{2}$ \\ $\left({ }^{1}\right.$ College of Life Science and Chemistry, Tianshui Normal University, Tianshui 741001, Gansu Province, P. R. China; \\ ${ }^{2}$ College of Chemistry and Chemical Engineering, Northwest Normal University, Lanzhou 730070, P. R. China)
}

\begin{abstract}
The mechanism of the $\mathrm{O}_{2}$ activation by the protocatechuate 3,4-dioxygenase was investigated using density functional calculations. In the initial complex, the ultrafast formation of the sextet ${ }^{6} 1$ was probably the result of electron-exchange-induced intersystem crossing, and $\mathrm{Fe} d_{z}: \mathrm{O}_{2} \pi^{*}(z)$ was the dominant exchange pathway, with an overlap of $S_{i j}=\left\langle d_{z^{2}} \alpha \mid \pi^{*}(z) \beta\right\rangle=0.3758$ at an $\mathrm{Fe}-\mathrm{O}$ bond length of $0.2487 \mathrm{~nm}$. Two coexisting effects, electron spin exchange coupling and spin-orbit coupling (SOC) in the sextet ${ }^{6} 1$, are responsible for formation of the quartet state ${ }^{4} 1$ from the sextet ${ }^{6} 1$. The exchange interaction competes with the SOC interaction as a driving force for spin conversion. The calculated results show that the latter is the dominant factor, because of the larger SOC constant $\left(353.16 \mathrm{~cm}^{-1}\right)$. In cleavage of the $\mathrm{O}-$ $O$ bond, electron transfer from the protocatechuate (PCA) highest occupied molecular orbital (HOMO) plays a vital role. The Fe center of the non-heme enzyme is a buffer to transfer an electron pair from the $\mathrm{PCA}$ HOMO to $\mathrm{O}_{2}$.
\end{abstract}

Key Words: Protocatechuate 3,4-dioxygenase; Spin-orbit coupling; Zero-field splitting; Reaction mechanism

\section{Introduction}

The mononuclear non-heme iron enzymes are an important group with a diverse range of chemical reactions including dioxygenation, hydroxylation, ring closure, oxidative desatura-

Received: February 27, 2013; Revised: June 3, 2013; Published on Web: June 4, 2013.

"Corresponding author. Email: lvling100@163.com; Tel: +86-18793855301.

The project was supported by the National Natural Science Foundation of China (21263022) and University Research Fund of Gansu Province

Financial Department, China.

国家自然科学基金(21263022)和甘肃省财政厅高校科研项目基金资助

C. Editorial office of Acta Physico-Chimica Sinica 
tion, and aromatic ring cleavage. ${ }^{1}$ Within this broad class, the oxygen-activating enzymes are one of the most extensively studied fields. ${ }^{1-4}$ Most non-heme oxygenases catalyze $\mathrm{O}_{2}$ activation using a high spin $\mathrm{Fe}(\mathrm{II})$ site through a redox process that also involves the substrate to provide the required number of electrons. By contrast, a small group of non-heme iron enzymes perform a high spin $\mathrm{Fe}(\mathrm{III})$ site to activate substrate for direct attack by $\mathrm{O}_{2}$, such as the lipoxygenases and intradiol dioxygenases.

Protocatechuate 3,4-dioxygenase (3,4-PCD) is one of the intradiol dioxygenase family. It catalyzes the ring cleavage of protocatechuate (PCA) to form $\beta$-carboxy-cis,cis-muconate, with the incorporation of both oxygen atoms from molecular oxygen. Based on different electronic descriptions of the enzyme-substrate (ES) complex, various mechanisms ${ }^{1}$ have been proposed for the initial $\mathrm{O}_{2}$ binding and activation steps, but they have not been definitively observed, therefore are not well understood. Mechanism for catechol ring cleavage by non-heme iron intradiol dioxygenases has been computationally investigated using density functional theory (DFT) by Borowski and Siegbahn. ${ }^{5}$ In 2003, Deeth and Bugg ${ }^{6}$ have also reported the studied results for the extradiol cleavage mechanism. But, in these investigations, the electronic structures and intersystem crossing processes of the initial $\mathrm{O}_{2}$ binding complexes have not been discussed in detail.

However, we know that the chemistry of transition metals and their compounds (especially, metal-containing enzymes complexes such as cytochromes P450 and non-heme iron) is strongly influenced by the availability of multiple low-lying electronic states in these species. ${ }^{7-9}$ Shaik and co-workers ${ }^{10}$ have proposed that multiple spin states play an important role in these reactions, with many of them involving what they have called "two-state reactivity (TSR)". The initial complexes of the triplet ground state $\mathrm{O}_{2}(S=1)$ bound to $\mathrm{Fe}(\mathrm{III})(S=5 / 2)$ of 3,4 -PCD-PCA can have a total spin of $S=7 / 2, S=5 / 2$, or $S=3 / 2$, and form the different spin state complexes. This means that the reactions should involve spin-conserving and spin-inversion processes. These will result in the complexity of the reaction mechanism.

Therefore, detailed analyses of electronic structures and intersystem crossing processes in the initial complexes are very important in order to better understand the $\mathrm{O}_{2}$ activation mecha- nism. In present paper we computed electronic exchange coupling $(J)$ and zero-field splitting (ZFS) using qausi-restricted theory at the DFT level ${ }^{11}$ implemented with the program ORCA. ${ }^{12}$ Then spin-orbit coupling (SOC) matrix elements were obtained by the approximate one-electron spin-orbit Hamiltonian. ${ }^{13}$ Some very meaningful conclusions have been obtained by these calculations.

\section{Computational details \\ 2.1 Step of the system}

The activate-site geometric structure of 3,4-PCD-PCA was obtained from the averaged crystallographic coordinate of $P$. putida 3,4-PCD complex with PCA (PDB code 3PCA). ${ }^{14}$ Hydrogen atoms were placed at standard bond lengths and angles. Optimize calculations were performed on a model of the triplet state $\mathrm{O}_{2}(S=1)$ bound to 3,4-PCD-PCA involving two Me-imidazoles to model His460 and His462, 4-Me-phenolate to model Tyr408, and a bidentate PCA in the fully deprotonated state, which is shown in Scheme 1.

Unrestricted calculations allow $\alpha$ and $\beta$ electrons to occupy orbitals with different spatial localizations and, can describe the spin localized on two different paramagnetic centers. Therefore, density functional theory calculations were performed using the Gaussian 03 program ${ }^{15}$ with spin-unrestricted functional U-BP86 with 10\% Hartree-Fock exchange and the Pople triple- $\zeta$ basis set, $6-311 \mathrm{G}(d)$ to optimize geometry of the active model described above. For comparison, calculations were also performed with the spin-unrestricted U-B3LYP functional with the LanL2DZ effective core potential basis set. Transition state structures were found to have one imaginary frequency, which correlated to the motion of the bond being broken or formed.

\subsection{Calculations of zero-field splitting (ZFS) tensor}

In the absence of nuclear spins and exchange interactions, the effective spin Hamiltonian of these interactions is usually written as

$$
H_{\text {spin }}=H_{\mathrm{ze}}+H_{\mathrm{zFs}}=\beta_{\mathrm{B}} B g S+S D S
$$

where $H_{\mathrm{ze}}$ is Zeeman effect operator, $H_{\mathrm{zFs}}$ is zero-field splitting operator, $\beta_{\mathrm{B}}$ is the Bohr magneton, $B$ is the magnetic flux density, $S$ is the effective spin operator, and $g$ and $D$ are the $g$-tensor and ZFS-tensor, respectively. $H_{\text {spin }}$ acts on the basis functions $|S M\rangle$ with $M=S, S-1, \cdots,-S$. If we choose a coordinate
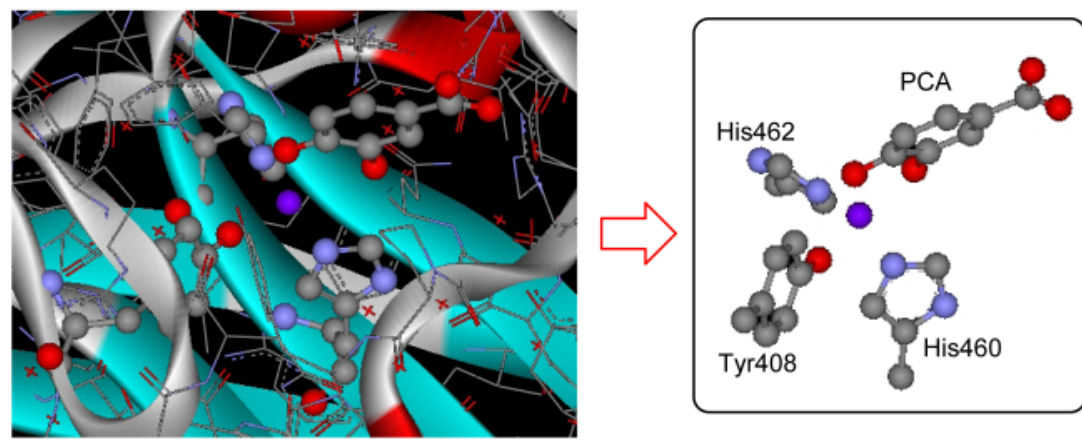

Scheme 1 Active-site geometric structures of 3,4-PCD-PCA (PDB code 3PCA) 
system that diagonalizes $D, H_{\mathrm{zFS}}$ can be rewritten:

$$
\begin{aligned}
& H_{\mathrm{ZFS}}=D\left[S_{z^{2}}-1 / 3 S(S+1)\right]+E\left(S_{x^{2}}-S_{y^{2}}\right) \\
& D=D_{z z}-1 / 2\left(D_{x x}+D_{y y}\right) ; \quad E=1 / 2\left(D_{x x}-D_{y y}\right)
\end{aligned}
$$

Thus, the ZFS is uniquely defined by the parameters $D$ and $E$ and the tensor orientation. Typically, $D$ and $E / D$ are given in a coordinate system that fulfils the following condition:

$$
0 \leq E / D \leq \frac{1}{3}
$$

DFT calculations of the ZFS were carried out using qausirestricted theory ${ }^{11}$ by the ORCA program. ${ }^{12}$ Previous studies ${ }^{11}$ have investigated the dependences of the $g$-tensor and ZFS on the exchange-correlation functional. Thus, in this work, we obtain ZFS parameters ( $D$ and $E$ ) from additional single-point calculations using 6-311G $(d)$ basis sets and the BP86 GGA functional. We use a recently described SOC operator that efficiently implements a spin-orbit mean-field (SOMF) method. ${ }^{16}$

\subsection{Spin-orbit coupling and exchange coupling calculations}

Exchange coupling constants $(J)$ are calculated using the Yamaguchi formula, which covers consistently the whole range of situations from the strong to the weak exchange coupling limit: ${ }^{17,18}$

$$
J=-\frac{E_{\mathrm{HS}}-E_{\mathrm{BS}}}{\left\langle S^{2}\right\rangle_{\mathrm{HS}}-\left\langle S^{2}\right\rangle_{\mathrm{BS}}}
$$

where $E_{\mathrm{HS}}$ and $E_{\mathrm{BS}}$ account for the energies of the high-spin and broken-symmetry states, respectively; $\left\langle S^{2}\right\rangle_{\text {HS }}$ and $\left\langle S^{2}\right\rangle_{\text {BS }}$ denote the spin angular momentum calculated in the high-spin and broken-symmetry solutions, respectively. These calculations were performed with a development version of the ORCA program. ${ }^{12}$

In addition, in order to obtain the more detailed SOC matrix elements, the SOC calculations of the sextet and quartet states were studied using the approximate one-electron spin-orbit Hamiltonian $\left(h_{i}\right)^{13}$ given in Eq.(4):

$$
H_{\mathrm{SO}}=\frac{\alpha^{2}}{2} \sum_{i} \sum_{k}\left(\frac{Z_{k}^{*}}{r_{i k}^{3}}\right)\left(S_{i} \cdot L_{i k}\right)=\sum_{i} h_{i}\left(Z^{*}\right), \frac{\alpha^{2}}{2}=\frac{e^{2} h}{4 \pi m_{e}^{2} c^{2}}
$$

where $L_{i k}$ and $S_{i}$ are the orbital and spin angular momentum operators for electron $i$ in the framework of the nuclei indexed $k$, respectively, and $Z_{k}^{*}$ is the effective nuclear charge, $r_{i k}$ is the distance of between each electron samples $(i)$ and all nuclei $(k)$. One-electron SOC calculations were carried out using the GAMESS program. ${ }^{19}$

\section{Results and discussion}

\subsection{Initial complex electronic states}

The optimized geometries and energetic data for the octet, sextet, and quartet electronic states are depicted in Fig.1, in order to keep the discussion more simple, the goal complex, denoted as 1 , is initially formed as 3,4-PCD-PCA and $\mathrm{O}_{2}$ collide end-on with each other, where the superscripts denote the spin multiplicities $\left({ }^{8} 1,{ }^{6} 1\right.$, and $\left.{ }^{4} 1\right)$.

\subsubsection{Octet state $(S=7 / 2)$}

The singly occupied molecular orbitals (SOMOs) of ${ }^{8} 1$ are plotted in Fig.2 and It clearly shows that $\mathrm{O}_{2}$ has two unpaired electrons, $\mathrm{O}_{2} \pi^{*}(y)$ and $\pi^{*}(z)$, while five others reside on the ferrous $\mathrm{Fe}(\mathrm{III})$ center, namely occupy five $d$ orbitals, thus forming a high-spin (HS) $\mathrm{Fe}(\mathrm{III})(S=5 / 2)$ combined ferromagnetically with a triplet $\mathrm{O}_{2}(S=1)$. Therefore the octet ${ }^{8} 1$ is approximately understood as van der Waals complex, which involves $\mathrm{O}_{2}$ in a end-on orientation with a long $\mathrm{Fe}-\mathrm{O}$ bond length of about $0.2558 \mathrm{~nm}$ at the U-B3LYP/LanL2DZ level $(0.2487 \mathrm{~nm}$ for U-BP86/6-311G*), as shown in Fig.1.

\subsubsection{Sextet state $(S=5 / 2)$}

We obtained a sextet ${ }^{6} 1$ complex as shown in Fig.1. Compared with ${ }^{8} 1$, the $\mathrm{Fe}-\mathrm{O}$ bond length was shortened to 0.1906 $\mathrm{nm}$ for U-BP86/6-311G*, while the $\mathrm{O}-\mathrm{O}$ bond length was increased to $0.1281 \mathrm{~nm}$. These show that $\mathrm{O}_{2}$ and Fe center has obvious chemically bonded effect in the sextet ${ }^{6} 1$ complex. The electronic structure of ${ }^{6} 1$ is schematically shown in Fig. 3. It is seen that $\mathrm{O}_{2}$ is a superoxide, having a singly occupied $\mathrm{O}_{2} \pi^{*}(y)$, which is vertical to the $\mathrm{Fe}-\mathrm{OO}$ plane, while the other doubly occupied $\pi^{*}(z)$ [ $\pi^{*}(z)$ is in $\mathrm{Fe}-\mathrm{OO}$ plane] forms a two-electron bond with the iron $d_{z^{2}}$ orbital. ${ }^{6} 1$ has an ferromagnetically coupling of the $S=1 / 2$ superoxo anion $\mathrm{O}_{2}^{-}$with $S=2 \mathrm{Fe}$ (IV) center. ${ }^{8} 1$ as zero reference, the U-BP86 calculated relative energy of ${ }^{6} 1$ is $-12.14 \mathrm{~kJ} \cdot \mathrm{mol}^{-1}$.

\subsubsection{Quartet state $(S=3 / 2)$}

For the quartet state ${ }^{4} 1$, in contrast with the sextet ${ }^{6} 1$ case, one pronounced difference is that the Fe(IV) center has two singly occupied orbitals $\left(d_{x y}\right.$ and $\pi_{x z}^{*}, S=1$ ), that is, ${ }^{4} 1$ differs from ${ }^{6} 1$ by a spin-flip of the a singly occupied orbital from $\sigma_{x^{2}-y^{2}}$ to $d_{y z}$, consequently, $d_{y z}$ is doubly occupied. $\mathrm{O}_{2}(S=1 / 2)$ is still a superoxide with a singly occupied $\pi^{*}(y)$ and a doubly occupied $\pi^{*}(z)$ orbitals, and coupled ferromagnetically with the Fe(IV) $(S=1)$, thus formed an intermediate-spin $S=3 / 2$ complex, ${ }^{4} 1$. The U-BP86 calculated energy difference is $9.21 \mathrm{~kJ} \cdot \mathrm{mol}^{-1}$ relative to the ${ }^{6} 1$ state.

\subsection{Intersystem crossing process of initial complexes}

As has been already discussed before, the triplet $\mathrm{O}_{2}(S=1)$ mixes with $\mathrm{Fe}(\mathrm{III})(S=5 / 2)$ resulting in the different spin states with a total spin of $S=7 / 2, S=5 / 2$, or $S=3 / 2$, and smaller energy differences among them. Moreover, we found that the spin states from the octet to quartet state are changed with the decrease of the $\mathrm{Fe}-\mathrm{O}$ bond length (from 0.2487 to $0.1895 \mathrm{~nm}$ at the U-BP86/6-311G* level), and the sextet ${ }^{6} 1$ is the most stable as shown in Fig.4. In this respect, the interesting question is raised: how is the intersystem crossing of the different spin states happened?

\subsubsection{Exchange coupling $(J)$}

Let us turn to discuss this question now, starting from the octet state ${ }^{8} 1$. To identify some main atomic orbital interactions, the main antiferromagnetic orbital interactions of ${ }^{8} 1$ were also inferred from overlaps calculated from the broken-symmetry wave function (U-BP86/6-311G*), the results calculated are plotted in Fig.5. In general, the broken-symmetry orbitals of 


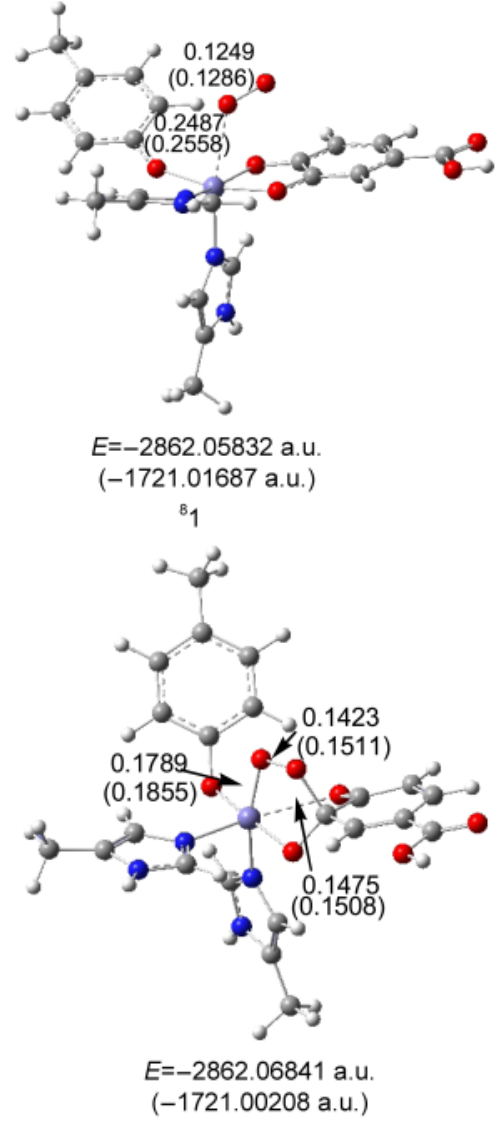

${ }^{4} 2$
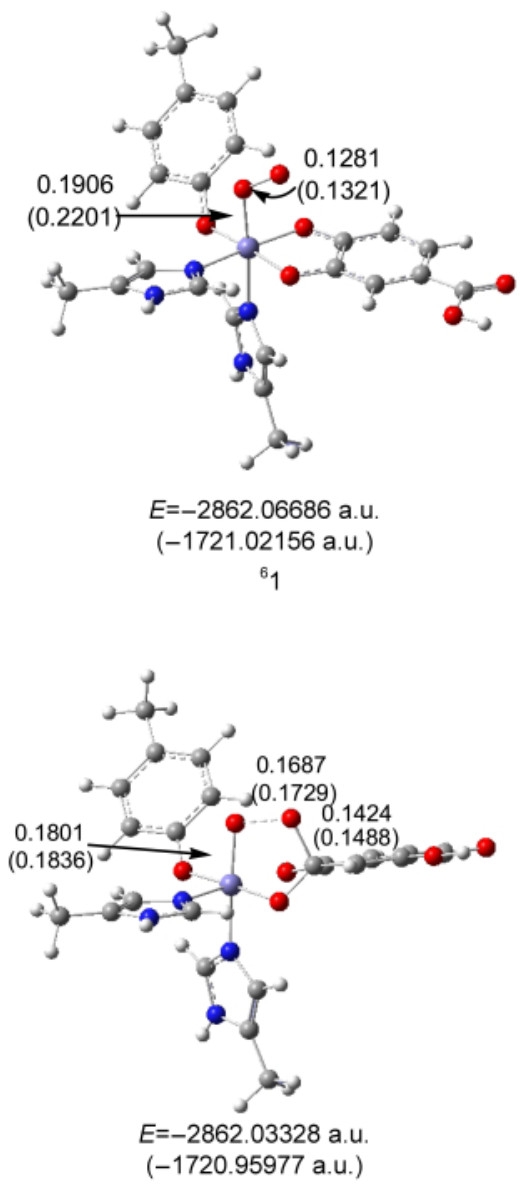

${ }^{4} \mathrm{TS}$
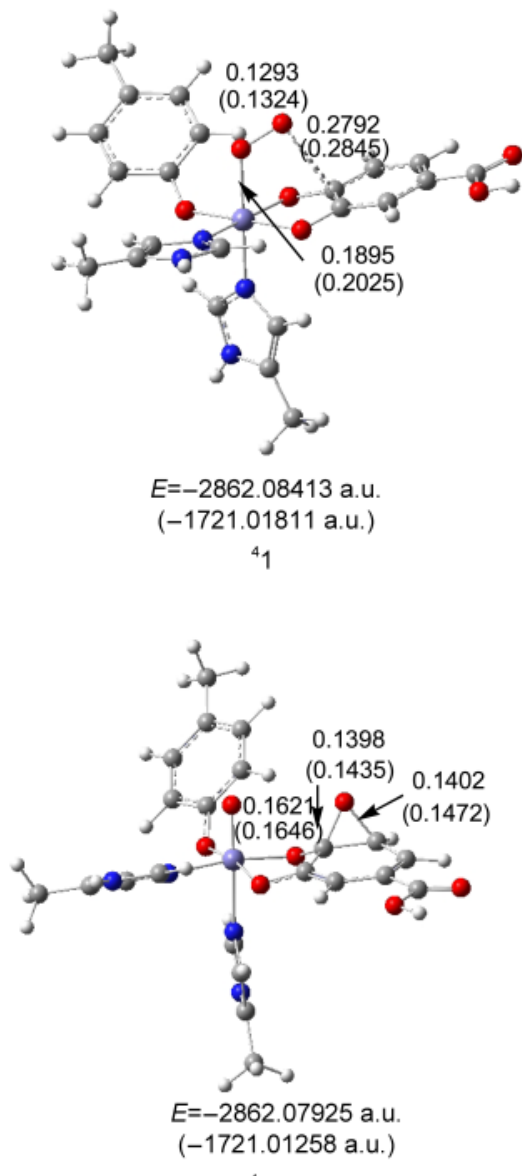

${ }^{4} \mathrm{P}$

Fig.1 Selected bond lengths $(\mathrm{nm})$ and energies (listed under the structures) obtained from the key point optimizations at the U-BP86/6-311G $(d)$ and U-B3LYP/LanL2DZ levels (in the parentheses)

$2^{4}$ is the second intermediate, TS is transition state, $\mathrm{P}$ is product.

different spin indexes (i.e., $\alpha$ or $\beta$ ) are not orthogonal to each other and are localized on $\mathrm{Fe}\left(\phi_{i} \alpha\right)$ or $\mathrm{O}_{2}\left(\phi_{j} \beta\right)$. Therefore, to this extent, the overlaps $S_{i j}=\left\langle\phi_{j} \alpha \mid \phi_{j} \beta\right\rangle$ are intimately related to the strength of antiferromagnetic spin coupling. As seen from Fig.5, the overlap between the localized $\mathrm{Fe} d_{z^{2}}$ and $\mathrm{O}_{2} \pi^{*}(z)$, $129 \alpha$ and $129 \beta$, is considerably better and $S_{i j}=\langle 129 \alpha \mid 129 \beta\rangle=$ 0.3758 at the $\mathrm{Fe}-\mathrm{O}$ distance of $0.2487 \mathrm{~nm}$. The low spin cou-

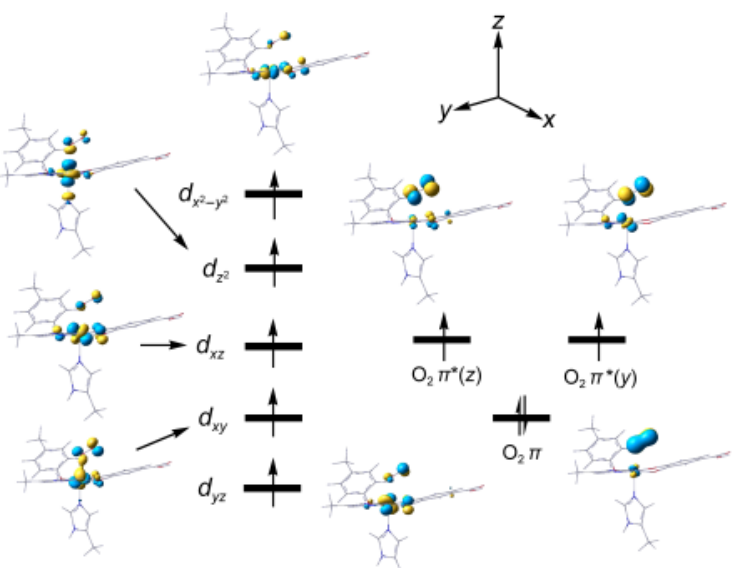

Fig.2 Electronic configuration of the octet state ${ }^{8} 1$ pling between $129 \alpha$ and $129 \beta$ electron pair is therefore strong enough to lead to a $\mathrm{Fe}-\mathrm{O}$ bonding. By contrast, the other overlap $S=\langle 130 \alpha \mid 130 \beta\rangle$ of the Fe $d_{y z}$ and $\mathrm{O}_{2} \pi^{*}(y)$ is much weaker and $S_{i j}=0.0868$. Thus the magnitude of the overlaps strongly suggested that the first $\mathrm{Fe} d_{z^{2}}: \mathrm{O}_{2} \pi^{*}(z)$ was clearly dominant ex-

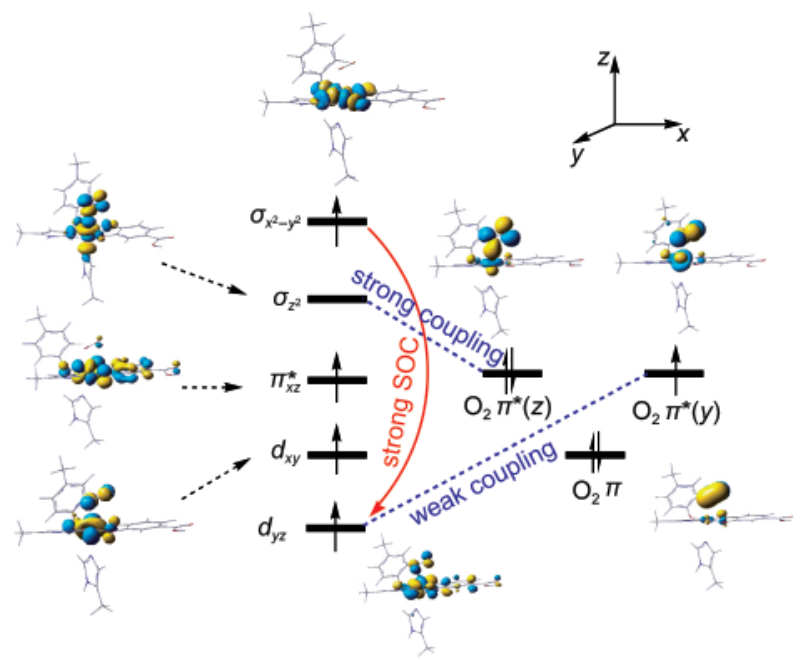

Fig.3 Occupied active natural orbitals of ${ }^{6} 1$ at the BP86/6-311G $(d)$ level 


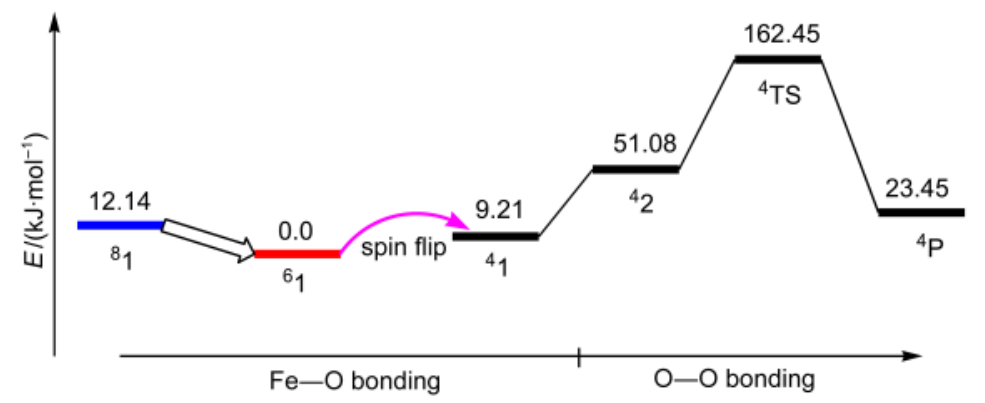

Fig.4 Schematic representation of calculated reaction paths at the B3LYP/LanL2DZ level

change pathway, whereas $\mathrm{Fe} d_{y z}: \mathrm{O}_{2} \pi^{*}(y)$ contributed to a lesser extent and was fairly comparable. At the same time, we found that the overlaps are increased with the decrease of the $\mathrm{Fe}-\mathrm{O}$ bonding, and the change of the exchange coupling constant $J$ is very similar to that of the overlaps, the $J$ values are increased from -20.8 to $-81.4 \mathrm{~cm}^{-1}$ with the distance of the $\mathrm{Fe}-\mathrm{O}$ bonding from 0.2487 to $0.2200 \mathrm{~nm}$. These calculations provided a detailed approximation to antiferromagnetism induced by Fe $d_{z^{2}}$ : $\mathrm{O}_{2} \pi^{*}(z)$ delocalization.

Therefore the formation of ${ }^{6} 1$ from ${ }^{8} 1$ is most likely due to the electron exchange induced enhanced intersystem crossing (EISC).$^{20}$ Certainly, for antiferromagnetic exchange, the energy gap between ${ }^{6} 1$ and ${ }^{8} 1$ is also very important. The energy gap between these two states is about $12.14 \mathrm{~kJ} \cdot \mathrm{mol}^{-1}$, one might expect that EISC would be faster. The electron exchange interaction between $\mathrm{Fe}$ (III) $(S=5 / 2)$ and $\mathrm{O}_{2}(S=1)$ serves as the first-order perturbation that drives EISC. The magnitude of this perturbation, and thus the overall intersystem crossing rate, depends strongly on the electronic overlap between the orbitals that contribute to the singly occupied molecular orbitals (SOMO) of the $\mathrm{Fe}(\mathrm{III})(S=5 / 2)$ center and $\mathrm{O}_{2}(S=1)$.
In addition, antiferromagnetic exchange coupling can lead to the partly forming of ${ }^{4} 1$ due to the weaker overlap $S=\langle 130 \alpha \mid 130 \beta\rangle$. We also noted that the quartet wave function can have some admixture of the sextet wave function. The effect was more noticeable, the expectation value of the total spin operator after annihilation, $\left\langle S^{2}\right\rangle=5.47$ was far from the value expected for a pure quartet state, $\left\langle S^{2}\right\rangle=3.75$. Based on the following expressions for the spin expectation values of the contaminated quartet state: ${ }^{18}$

$$
\begin{aligned}
& \left(S_{4}^{\mathrm{BS}}\right)^{2} \approx C_{6}^{2} S_{6}\left(S_{6}+1\right)+C_{4}^{2} S_{4}\left(S_{4}+1\right) \\
& C_{6}^{2}+C_{4}^{2} \approx 1
\end{aligned}
$$

where $C$ is the configuration coefficient, $S$ is the total spin operator, subscripts 6 and 4 denote sextet state and quartet state, respectively. From Eq.(5) we obtain the contribution of the quartet state which is about $67 \%$ of the mixing states. This reason is that a second-order term of ZFS, SOC) introduces some angular momentum into the sextet state. These mechanisms above are described in Fig.3.

\subsubsection{Zero-field splitting (ZFS)}

The net effects for ZFS are to introduce a splitting of the $2 S+$ 1 , in the absence of an external magnetic field. This will main-
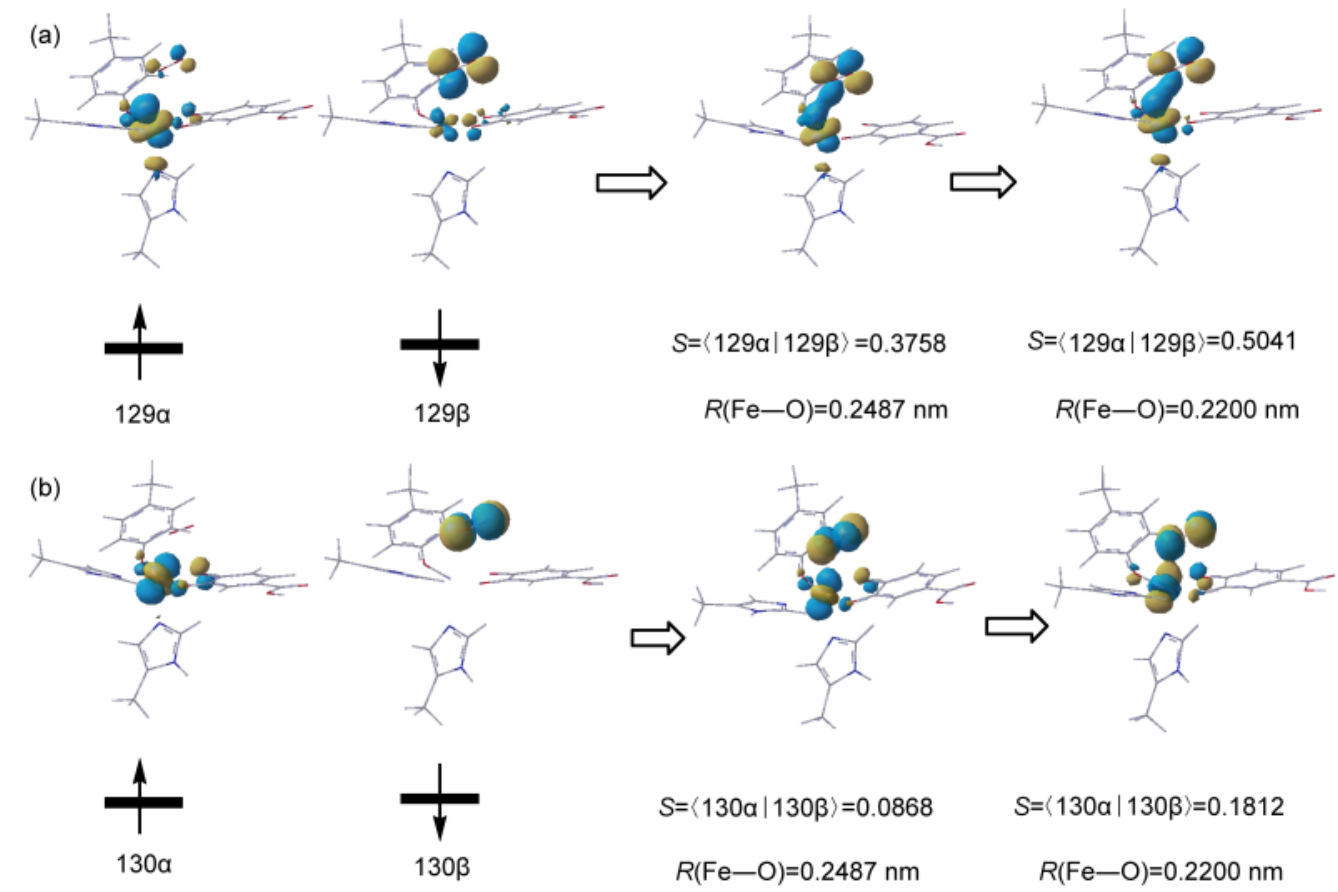

Fig.5 Pathways of the exchange coupling from ${ }^{8} 1$ to $^{6} 1$ or $^{4} 1$ 
ly attribute to the ZFS's two contributions: ${ }^{11}$ (a) a first-order term, the direct dipolar spin-spin (SS) interaction between pairs of electrons and (b) a second-order term, arising from SOC. Thus an analysis and interpretation of the ZFS is imperative in this study.

BP86 calculations were performed to determine the signs of the $D$ and $E$ values for ${ }^{6} 1$. The ZFS parameters $\left[D=D_{z z}-1 / 2\left(D_{x x}+\right.\right.$ $\left.\left.D_{y y}\right), E=1 / 2\left(D_{x x}-D_{y y}\right)\right]$ are calculated, $D=+8.589 \mathrm{~cm}^{-1}$ and $E / D=+$ 0.068 . The more detailed results of the calculated ZFS using the quasi-restricted DFT method are shown in Table 1. From the results in Table 1, the major contribution arises from the second-order SOC contribution (around $97.5 \%$ of $D$ ), while the SS contributions are essentially negligible. Concerning the SOC part, it contains four significant contributions: $\alpha \rightarrow \alpha(D=$ $\left.0.332 \mathrm{~cm}^{-1}\right), \beta \rightarrow \beta\left(D=0.240 \mathrm{~cm}^{-1}\right), \alpha \rightarrow \beta\left(D=8.138 \mathrm{~cm}^{-1}\right)$, and $\beta \rightarrow \alpha\left(D=-0.336 \mathrm{~cm}^{-1}\right)$. However the major contribution comes from the $\alpha \rightarrow \beta$ spin-flip excitation. This excitation contributes around $77.2 \%$ of the $D_{\mathrm{SOC}}$ value, corresponding to the spin-pairing $\Delta S=-1$ (i.e., sextet $\rightarrow$ quartet) transitions. The second larger SOC contribution arises from the $\beta \rightarrow \alpha$ spin-raising $\Delta S=+1$ (sextet $\rightarrow$ octet) excitation (around $4 \%$ of $D_{\mathrm{SOC}}$ ) corresponding to ligand-to-metal charge-transfer transitions. The remaining two SOC contributions come from the spin allowed $\Delta S=0$ (sextet $\rightarrow$ sextet) ligand-field excitations and have usually been solely held responsible for the ZFS. However, these contributions are only around $7 \%$ compared to the $D_{\mathrm{SOC}}$ value.

The $\Delta S=-1$ state (sextet is flipped to quartet state) is found to make significant contributions to $D_{\mathrm{SOC}}$, with the primary contribution arising from the single-determinant spin-paired states within the single occupied sets of orbitals, $\sigma_{x^{2}-y^{2}}, \pi_{x z}^{*}, d_{x y}$, and $d_{y z}$ (see Fig.3). Namely, $D_{x x}\left(\sigma_{x^{2}-y^{2}} \uparrow \rightarrow d_{y z} \downarrow\right), D_{y y}\left(\sigma_{x^{2}-y^{2}} \uparrow \rightarrow \pi_{x z}^{*} \downarrow\right)$, and $D_{z z}\left(\sigma_{x^{2}-y^{2}} \uparrow \rightarrow d_{x y} \downarrow\right)$, vital spin-flip excitation. Applying the angular momentum operator to this four orbitals generates three nonzero SOC integrals: $\left\langle\sigma_{x^{2}-y^{2}}\left|l_{x}\right| d_{y z}\right\rangle,\left\langle\sigma_{x^{2}-y^{2}}\left|l_{y}\right| \pi_{x z}^{*}\right\rangle$, and $\left\langle\sigma_{x^{2}-y^{2}}\right|$ $l_{z}\left|d_{x y}\right\rangle$ which make the largest contribution to the SOC and lead to the larger values of $D_{x x}\left(=8.8 \mathrm{~cm}^{-1}\right), D_{z z}\left(=16.8 \mathrm{~cm}^{-1}\right)$, and $D_{y y}\left(=7.6 \mathrm{~cm}^{-1}\right)$. It identifies the origin of large ZFS as spinorbit coupling to low-lying $\Delta S=-1$ state, and shows that the quartet ${ }^{4} 1$ can arise from normal spin-orbit induced intersystem crossing as compared with the weaker exchange coupling.

\subsubsection{Spin-orbit coupling}

Table 1 Contributions to the calculated zero-field splitting between spin-orbit coupling and spin-spin for the sextet state

\begin{tabular}{|c|c|c|c|c|}
\hline Method & \multicolumn{2}{|c|}{ Individual contributions } & $D / \mathrm{cm}^{-1}$ & $E / \mathrm{cm}^{-1}$ \\
\hline BP86-QR-SOMF/ & spin-orbit-coupling & $\alpha \rightarrow \alpha$ & 0.332 & 0.020 \\
\hline \multirow[t]{7}{*}{ TZVP } & & $\beta \rightarrow \beta$ & 0.240 & 0.121 \\
\hline & & $\alpha \rightarrow \beta$ & 8.138 & 0.192 \\
\hline & & $\beta \rightarrow \alpha$ & -0.336 & 0.174 \\
\hline & & total & 8.374 & 0.549 \\
\hline & spin-spin & coulomb & 0.180 & 0.013 \\
\hline & & exchange & 0.035 & 0.018 \\
\hline & & total & 0.215 & 0.031 \\
\hline
\end{tabular}

Based on the above analysis, the sextet ${ }^{6} 1$ from ${ }^{8} 1$ is fast formed via EISC, following the system will well change its spin multiplicities from the sextet state to the quartet ground state ${ }^{4} 1$ by $\mathrm{SOC}$ in the $\mathrm{O}_{2}$ gradual approach to $\mathrm{Fe}$ center process. However, a spin-forbidden transition requires an effect of SOC that provides a major mechanism for the intersystem crossing process. ${ }^{21-24}$ Therefore, we must inspect the orbital relationships which promote the SOC matrix elements.

The ROHF orbitals for the construction of the quartet and sextet CASCI wave functions to be used in the SOC evaluation have been generated by the sextet ROHF calculation. At the $\operatorname{CAS}(7,6)$ level the dominant determinants of the MCSCF wave functions have configuration interaction (CI) coefficients of 0.98 and 0.17 for the sextet and 0.86 and -0.48 for the quartet state (configurations of less than 0.1 have not been listed). Thus the permissible approximation of the sextet wave function by a single configuration (0.98) enables us to analyze the SOC matrix elements. The quartet configuration is described as shown in Eq.(6),

$$
\left.\left|{ }^{4} \psi\right\rangle=0.86^{4}\left|{ }^{4} d_{y z}^{2} \pi^{*}(y)^{1} d_{x y}^{1} \pi_{x z}^{* 1}\right\rangle-\left.0.48\right|^{4} d_{y z}^{1} \pi^{*}(y)^{1} d_{x y}^{2} \pi_{x z}^{* 1}\right\rangle
$$

Nonzero elements of the $p$-components $(p=x, y, z)$ of the SOC matrix, $\left\langle{ }^{6} \psi\left(M_{\mathrm{s}}\right)\left|H_{\mathrm{SO}}\right|{ }^{4} \psi\left(M_{\mathrm{s}}\right)\right\rangle_{p}$, are always proportional to the function, $F_{p}$, as given in Eq.(7).

$F_{p}=2^{-1 / 2} \times 0.86\left\langle\sigma_{x^{2}-y^{2}}\left|H_{\mathrm{SO}, k}\right| d_{y z}\right\rangle-2^{-1 / 2} \times 0.48\left\langle\sigma_{x^{2}-y^{2}}\left|H_{\mathrm{SO}, k}\right| d_{x y}\right\rangle(7)$

To further understand the efficient SOC, it is very important that the SOC matrix elements $\left\langle\sigma_{x^{2}-y^{2}}\left|H_{\mathrm{SO}, k}\right| d_{y z}\right\rangle$ and $\left\langle\sigma_{x^{2}-y^{2}}\left|H_{\mathrm{SO}, k}\right| d_{x y}\right\rangle$ are discussed. Because the SOC constant $\left(\xi_{\mathrm{Fe}}\right)$ is an order of magnitude greater than that of oxygen, making it a reasonable approximation to consider only the Fe contribution when discussing spin-orbit mixing with quartet states. Thus

$$
\begin{aligned}
\langle\mathrm{SOC}\rangle= & \eta C_{0} C_{j 1} \sum_{p} \xi_{\mathrm{Fe}}\left\langle d_{x^{2}-y^{2}}\left|L_{\mathrm{Fe}, p}\right| d_{y z}\right\rangle\left\langle\theta_{2}\left|S_{p}\right| \theta_{4}\right\rangle+ \\
& \eta C_{0} C_{j 2} \sum_{p} \xi_{\mathrm{Fe}}\left\langle d_{x^{2}-y^{2}}\left|L_{\mathrm{Fe}, p}\right| d_{x y}\right\rangle\left\langle\theta_{2}\left|S_{p}\right| \theta_{3}\right\rangle
\end{aligned}
$$

where the $\eta$ term is the $M_{s}$-depended weight weighing factor, and $\theta=\alpha$ and /or $\beta$. In the present case, for the sextet state, the

\begin{tabular}{|c|c|c|c|c|}
\hline State 1 & State 2 & $\left\langle H_{\mathrm{so}}\right\rangle_{r}$ & $\left\langle S_{1}=5 / 2 ; M_{s 1}\left|H_{\mathrm{so}}\right| S_{2}=3 / 2 ; M_{s 2}\right\rangle$ & SOC \\
\hline \multirow[t]{12}{*}{${ }^{6} \mathrm{~A}$} & ${ }^{4} A$ & $r=z$ & $\left\langle M_{s 1}=3 / 2\left|H_{\mathrm{so}}\right| M_{s 2}=3 / 2\right\rangle=-86.17$ & 353.16 \\
\hline & & & $\left\langle M_{s 1}=1 / 2\left|H_{\mathrm{SO}}\right| M_{s 2}=1 / 2\right\rangle=-105.55$ & \\
\hline & & & $\left\langle M_{s 1}=-1 / 2\left|H_{\mathrm{so}}\right| M_{s 2}=-1 / 2\right\rangle=-105.55$ & \\
\hline & & & $\left\langle M_{s 1}=-3 / 2\left|H_{\mathrm{so}}\right| M_{s 2}=-3 / 2\right\rangle=-86.17$ & \\
\hline & & $r=x, y$ & $\left\langle M_{s 1}=5 / 2\left|H_{\mathrm{so}}\right| M_{s 2}=3 / 2\right\rangle=141.21 ; 44.23$ & \\
\hline & & & $\left\langle M_{s 1}=-5 / 2\left|H_{\mathrm{sO}}\right| M_{s 2}=-3 / 2\right\rangle=141.21 ;-44.23$ & \\
\hline & & & $\left\langle M_{s 1}=3 / 2\left|H_{\mathrm{so}}\right| M_{s 2}=1 / 2\right\rangle=109.38 ; 34.26$ & \\
\hline & & & $\left\langle M_{s 1}=-3 / 2\left|H_{\mathrm{so}}\right| M_{s 2}=-1 / 2\right\rangle=109.38 ;-34.26$ & \\
\hline & & & $\left\langle M_{s 1}=1 / 2\left|H_{\mathrm{SO}}\right| M_{s 2}=-1 / 2\right\rangle=77.34 ; 24.22$ & \\
\hline & & & $\left\langle M_{s 1}=-1 / 2\left|H_{\mathrm{sO}}\right| M_{s 2}=1 / 2\right\rangle=77.34 ;-24.22$ & \\
\hline & & & $\left\langle M_{s 1}=-1 / 2\left|H_{\mathrm{so}}\right| M_{s 2}=-3 / 2\right\rangle=44.65 ; 13.99$ & \\
\hline & & & $\left\langle M_{s 1}=1 / 2\left|H_{\mathrm{SO}}\right| M_{s 2}=3 / 2\right\rangle=44.65 ; 13.99$ & \\
\hline
\end{tabular}
fundamental open-shell configuration has one dominant coeffi-

Table 2 Calculated SOC matrix elements $\left(\mathrm{cm}^{-1}\right)$ in the ${ }^{4} 1$ structure 


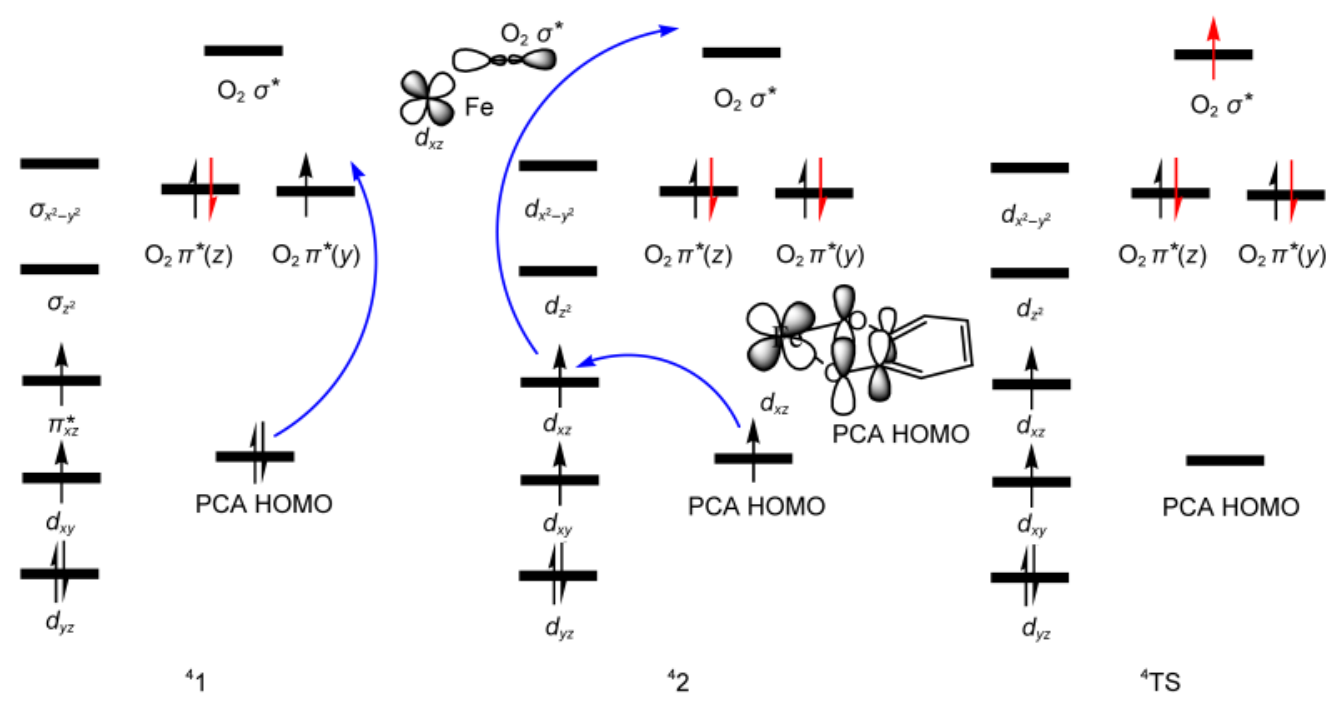

Fig.6 Electronic evolution during the $\mathrm{O}-\mathrm{O}$ bond cleavage

cient, i.e., $C_{0}=0.98 \approx 1$, while coefficients of quartet states are $C_{j 1}=0.86$ and $C_{j 2}=-0.48$, respectively.

Therefore:

$$
\begin{aligned}
\left\langle d_{x^{2}-y^{2}}\left|L_{\mathrm{Fe}, p}\right| d_{y z}\right\rangle\left\langle\theta_{2}\left|S_{p}\right| \theta_{3}\right\rangle= & \left\langle d_{x^{2}-y^{2}}\left|L_{\mathrm{Fe}, x, y}\right| d_{y z}\right\rangle\left\langle\alpha\left|S_{x, y}\right| \beta\right\rangle+ \\
& \left\langle d_{x^{2}-y^{2}}\left|L_{\mathrm{Fe}, z}\right| d_{y z}\right\rangle\left\langle\alpha\left|S_{z}\right| \alpha\right\rangle
\end{aligned}
$$

Here, only the first term is nonzero value, the electron shift from $d_{x^{2}-y^{2}}$ to $d_{y z}$ creates an non-zero angular momentum in the $L_{x}$-direction, resulting in the larger SOC matrix elements as listed in Table 2. The second term is zero value due to the mismatch for the $d_{x^{2}-y^{2}} \rightarrow d_{y z}$ transition with $L_{z}$ angular momentum direction. Similarly, the larger $z$-direction SOCs come from $\left\langle d_{x^{2}-y^{2}}\right|$ $L_{\mathrm{Fe}, z}\left|d_{x y}\right\rangle\left\langle\alpha\left|S_{z}\right| \alpha\right\rangle$ coupling of the second term in Eq.(9). These analyses are in good agreement with the SOC calculations of the approximate one-electron spin-orbit Hamiltonian (see Table 2) and $D$-tensor of $\mathrm{ZFS},{ }^{25}$ namely there exist the larger SOC matrix elements in $L_{x^{-}}$and $L_{z}$-directions, resulting in the SOC constant of $353.16 \mathrm{~cm}^{-1}$. These also further indicated that the significant contributions to $D$ arise from the $\Delta S=-1$ spin-flip transition, and the quartet state ${ }^{4} 1$ is produced by a spin-orbit coupling intersystem crossing.

\subsection{Cleavage process of the $\mathrm{O}-\mathrm{O}$ bond}

Structural parameters of the transition state $\left({ }^{4} \mathrm{TS}\right)$ on the quartet surface are collected in Fig.1. The $\mathrm{O}-\mathrm{O}$ bond cleavage pathway is shown in Fig.6. The $\mathrm{O}-\mathrm{O}$ bond activation takes place through a precursor intermediate ${ }^{4} 2$ and transition state $\left({ }^{4} \mathrm{TS}\right)$, to afford a cleavage product ${ }^{4} \mathrm{P}$. The ${ }^{4} \mathrm{TS}$ has the feature of partially broken $\mathrm{O}-\mathrm{O}$ bond $(0.1687 \mathrm{~nm})$, in which the activation barrier is $111.37 \mathrm{~kJ} \cdot \mathrm{mol}^{-1}$ with respect to ${ }^{4} 2 .{ }^{4} \mathrm{TS}$ is characterized as a transition structure by one imaginary frequency of $839.7 \mathrm{i} \mathrm{cm}^{-1}$, and the vibrational vector corresponds to the expected components of the reaction coordinate, i.e., breaking of the $\mathrm{O}-\mathrm{O}$ bond.

Schematic of the frontier molecular orbitals which participate in the three-electron transfer process upon the $\mathrm{O}-\mathrm{O}$ bond cleavage is shown in Fig.6. As can been seen from Fig.6, the doubly occupied PCA $\pi$ orbital is the HOMO in the complex, one electron from the doubly occupied PCA $\pi$ orbital can be donated directly to the $\mathrm{O}_{2} \pi^{*}$ orbital to form the distorted $\mathrm{O}-$ $\mathrm{C}_{\mathrm{PCA}}$ bond. Two electrons are transferred to the $\mathrm{O}_{2} \pi^{*}$ orbital generating a $\mathrm{Fe}(\mathrm{IV})$-peroxide. There is the strong covalent interaction between the PCA HOMO and Fe $d_{x z}$ orbitals in the $\alpha$ manifold, the second electron of the PCA HOMO is transferred to $\mathrm{Fe}$, at the same time the transfer of an $\mathrm{Fe} d$ electron into the $\mathrm{O}_{2} \sigma^{*}$ orbital to break the $\mathrm{O}-\mathrm{O}$ bond is accomplished. Finally, cleavage of the $\mathrm{O}-\mathrm{O}$ bond leads to formation of an $\mathrm{Fe}(\mathrm{IV})$-oxo. Therefore, the transfer of these three electrons (one $\alpha$ and two $\beta$ ) shows that the Fe center acts as a buffer to transfer an electron pair from PCA $\pi$ orbital to the triplet $\mathrm{O}_{2}$ in the spin forbidden reaction.

\section{Conclusions}

The $\mathrm{O}_{2}$ activating mechanism by non-heme iron enzyme, 3,4-PCD-PCA, has been studied using theoretical calculations. The electronic structure origins and intersystem crossing of the different spin states (a total spin of $S=7 / 2, S=5 / 2$, or $S=3 / 2$ ) were discussed by the broken-symmetry method and SOC mechanism. The octet state ${ }^{8} 1$ is stable with the aid of the electrostatic interaction, while the ultrafast formation of ${ }^{6} 1$ is most likely due to EISC. As for the formation of the quartet state ${ }^{4} 1$ from the sextet ${ }^{6} 1$, there coexist the two effects, electron spin exchange coupling and spin-orbit coupling in the sextet ${ }^{6} 1$. As a driving force of spin conversion the exchange interaction competes with spin-orbit coupling interaction. The calculated results show that the latter is the dominant factor due to the larger SOC constant $\left(353.16 \mathrm{~cm}^{-1}\right)$. The doublet ${ }^{2} 1$ optimization is failure using various DFT methods, all optimizations can not be converged due to the energy fluctuation.

The $\mathrm{O}-\mathrm{O}$ bond activation takes place through a precursor intermediate ${ }^{4} 2$ and transition state ( $\left.{ }^{4} \mathrm{TS}\right)$, to afford a cleavage 
product ${ }^{4} \mathrm{P}$. The electronic transfer of the PCA HOMO is the vital role for the cleavage of the $\mathrm{O}-\mathrm{O}$ bond. The Fe center of non-heme enzyme is a buffer to transfer an electron pair from PCA HOMO orbital to the $\mathrm{O}_{2}$ in the reaction.

\section{References}

(1) Pau, M. Y. M.; Davis, M. I.; Orville, A. M.; Lipscomb, J. D.; Solomon, E. I. J. Am. Chem. Soc. 2007, 129, 1944. doi: 10.1021/ja065671x

(2) Costas, M.; Mehn, M. P.; Jensen, M. P.; Que, L., Jr. Chem. Rev. 2004, 104, 939. doi: 10.1021/cr020628n

(3) Solomon, E. I.; Brunold, T. C.; Davis, M. I.; Kemsley, J. N.; Lee, S. K.; Lehnert, N.; Neese, F.; Skulan, A. J.; Yang, Y. S.; Zhou, J. Chem. Rev. 2000, 100, 235. doi: 10.1021/cr9900275

(4) Nam, W. Accounts Chem. Res. 2007, 40, 522. doi: 10.1021/ $\operatorname{ar} 700027 \mathrm{f}$

(5) Borowski, T.; Siegbahn, P. E. M. J. Am. Chem. Soc. 2006, 128, 12941. doi: $10.1021 / \mathrm{ja} 0641251$

(6) Deeth, R. J.; Bugg, T. D. H. J. Biol. Inorg. Chem. 2003, 8, 409.

(7) Fiedler, A.; Schroder, D.; Shaik, S.; Schwarz. H. J. Am. Chem. Soc. 1994, 116, 10734. doi: 10.1021/ja00102a043

(8) Yoshizawa, K.; Shiota, Y.; Yamabe, T. J. Chem. Phys. 1999, 111, 538. doi: 10.1063/1.479333

(9) Shaik, S.; Hirao, H.; Kumar, D. Accounts Chem. Res. 2007, 40, 532. doi: $10.1021 /$ ar600042c

(10) Schroder, D.; Shaik, S.; Schwarz, H. Accounts Chem. Res. 2000, 33, 139. doi: 10.1021/ar990028j

(11) Neese, F. J. Am. Chem. Soc. 2006, 128, 10213. doi: 10.1021/ ja061798a

(12) Neese, F. ORCA, version 2.8-20; Max-Planck Institute for Bioinorganic Chemistry: Mülheim an der Ruhr, Germany, 2010.

(13) Fedorov, D. G.; Koseki, S.; Schmidt, M. W.; Gordon, M. S. Int. Rev. Phys. Chem. 2003, 22, 551.
(14) Elgren, T. E.; Orville, A. M.; Kelly, K. A.; Lipscomb, J. D.; Ohlendorf, D. H.; Que, L., Jr. Biochemistry 1997, 36, 11504. doi: 10.1021/bi970691k

(15) Frisch, M. J.; Trucks, G. W.; Schlegel, H. B.; et al. Gaussian 03, Revision E.01; Gaussian Inc.: Pittsburgh, PA, 2003.

(16) Hess, B. A.; Marian, C. M.; Wahlgren, U.; Gropen, O. Chem. Phys. Lett. 1996, 251, 365. doi: 10.1016/0009-2614(96)00119-4

(17) Rodriguez, J. H.; McCusker, J. K. J. Chem. Phys. 2002, 116, 6253. doi: $10.1063 / 1.1461363$

(18) Rodriguez, J. H.; Wheeler, D. E.; McCusker, J. K. J. Am. Chem. Soc. 1998, 120, 12051. doi: 10.1021/ja980917m

(19) Schmidtm, M. W.; Baldridge, K. K.; Boatz, J. A.; Elbert, S. T.; Gordon, M. S.; Jensen, J. H.; Koseki, S.; Matsunaga, N.; Nguyen, K. A.; Su, S. J.; Windus, T. L.; Dupuis, M.; Motgomery, J. A. J. Comput. Chem. 1993, 14, 1347.

(20) Giacobbe, E. M.; Mi, Q.; Colvin, M. T.; Cohen, B.; Ramanan, C.; Scott, A. M.; Yeganeh, S.; Marks, T. J.; Ratner, M. A.; Wasielewski, M. R. J. Am. Chem. Soc. 2009, 131, 3700. doi: 10.1021/ja808924f

(21) Isobe, H.; Yamanaka, S.; Kuramitsu, S.; Yamaguchi, K. J. Am. Chem. Soc. 2008, 130, 132. doi: 10.1021/ja073834r

(22) Dede, Y.; Zhang, X.; Schlangen, M.; Schwarz, H.; Baik, M. H. J. Am. Chem. Soc. 2009, 122, 114.

(23) Lv, L. L.; Wang, Y. C.; Wang, Q.; Liu, H. W. J. Phys. Chem. C 2010, 114, 17610.

(24) Lü, L. L.; Wang, Y. C. Acta. Phys. -Chim. Sin. 2006, 22, 265. [吕玲玲, 王永成. 物理化学学报, 2006, 22, 265.] doi: 10.3866/ PKU.WHXB20060302

(25) Lü, L. L.; Zhu, Y. C.; Wang, X. F.; Zuo, G. F.; Zhao, S. R.; Guo, F.; Wang, Y. C. Chin. Sci. Bull. 2013, 58, 627. [吕玲玲, 朱元 成, 王小芳, 左国防, 赵素瑞, 郭 峰, 王永成. 科学通报, 2013, 58, 627.] doi: 10.1007/s11434-012-5316-7 